TRANSACTIONS OF THE

AMERICAN MATHEMATICAL SOCIETY

Volume 284, Number 1, July 1984

\title{
ON THE UNIVERSAL THEORY OF CLASSES OF FINITE MODELS ${ }^{1}$
}

BY

\author{
S. TULIPANI
}

\begin{abstract}
First order theories for which the truth of a universal sentence on their finite models implies the truth on all models are investigated. It is proved that an equational theory has such a property if and only if every finitely presented model is residually finite. The most common classes of algebraic structures are discussed.
\end{abstract}

0. Introduction. John T. Baldwin in a review of the book Selected papers of Abraham Robinson. Volume 1, posed the following problem: "For what first order theories $T$ does the truth of a universal sentence $\sigma$ on the finite models of $T$ imply that $\sigma$ is consequence of $T$ ?" Throughout this paper we will call such theories universally-finite. Baldwin's problem is suggested by Robinson's paper [19] where it is proved that the theory of Abelian groups and the theory of fields are universally-finite. See also Kueker [15].

We recall from well-known results [5] that a theory $T$ is universally-finite iff every model of $T$ can be embedded in an ultraproduct of finite models of $T$. However, such a characterization is difficult to handle even in simple cases. In this paper we look rather for a more useful characterization.

First, we confine our attention to equational theories (§2). Then, we show that to every theory $T$ in any language we can associate an equational theory $E$ in a language without relation symbols in such a way that Baldwin's problem for $T$ is reducible to Baldwin's problem of $E$ (Proposition 3 ).

The main theorem of this paper (see $\S 2$ ) proves that several statements are equivalent to the assertion that an equational theory $T$ is universally-finite. The most important are: "Every finitely presented model of $T$ is residually finite" and "Every quasi-identity true in all finite subdirectly irreducible models of $T$ is true in all subdirectly irreducible models of $T$ ".

We are convinced that our theorem provides a satisfactory characterization. In fact we get as an immediate consequence of it that the following varieties are universally-finite: Commutative unitary rings, commutative von Neumann regular rings with quasi-inverse as operation, lattices, $R$-modules, where $R$ is a finitely generated commutative unitary ring (this generalizes Robinson's result). On the other hand groups and unitary rings (cf. [6]) are examples of non-universally-finite varieties. Moreover, we characterize $(\S 4)$ the varieties of $R$-modules which are

Received by the editors June 20, 1983.

1980 Mathematics Subject Classification. Primary 03C13, 03C05; Secondary 03C60, 08C10.

Key words and phrases. Universal sentence, finite models, residually finite, finitely presented.

${ }^{1}$ This research is part of the program partially supported by CNR and MPI (60\%).

(C) 1984 American Mathematical Society $0002-9947 / 84 \$ 1.00+\$ .25$ per page 
universally-finite when $R$ is Noetherian, when $R$ is Artinian and when $R$ is a von Neumann commutative regular ring.

ACKNOWLEDGEMENTS. Thanks to some helpful comments of Professor G. Sabbagh and to a kind letter of Ch. Berline we could avoid a second lemma for Theorem 1.

The method of reduction to varieties in $\S 3$ was inspired by a very similar method used in $[\mathbf{2 2}, \S 9]$. However, as I learned from the Referee's report, I have to acknowledge that such a general method goes back to McKenzie (see [23]).

1. Notation and preliminary definitions. Theory always means first order theory in a language with equality. For notational simplicity we use capital italic letters $A, B, \ldots$ for structures as well as their basic sets. A structure for a language without relation symbols will be called an algebra. All members of a class of structures are assumed to be similar, i.e. of the same type.

If $K$ is a class of structures, we denote the class of finite members of $K$ by $K_{\text {fin }}$. We say that a class $K$ is universally-finite if every universal sentence in the language of $K$ which is true in all members of $K_{\text {fin }}$ is also true in all members of $K$. We say that a theory is universally-finite if the class of its models is universally-finite.

For the definitions of equational theories, of varieties, of identities and quasiidentities see [ $\mathbf{4}$ and $\mathbf{9}$ ]. We denote the usual operators of taking cartesian product, subalgebras, homomorphic images, reduced products, ultraproducts and finite direct product by $P, S, H, P_{R}, P_{U}, P_{\text {fin }}$, respectively. If $V$ is a variety, we denote the class of subdirectly irreducible members of $V$ by $V_{S I}$. An algebra of $V$ is called finitely presented if it is isomorphic to a quotient by a finitely generated congruence of a free algebra in $V$ of finite rank (cf. [17]). An algebra is called residually finite if it is the subdirect product of finite algebras.

All nonexplained notations will be standard. The reader is referred to $[\mathbf{5}, \mathbf{9}$ and 17] for notions of model-theory and universal algebra and to [1 and 21] for notions of algebra.

\section{Universally-finite varieties.}

LEMMA 1. Let $V$ be a variety and let $\sigma$ be an existential sentence in the language of $V$ which is consistent with $V$. Then, $\sigma$ holds in some finitely presented member of $V$.

Moreover, if $\sigma$ holds in $A$, where $A$ is a subdirect product of some family $\mathcal{F}$, then $\sigma$ holds in a direct product of a finite subfamily of $\mathcal{F}$.

PROOF. Without loss of generality we may assume that $\sigma$ is of the form $\exists x_{1} \ldots$ $\exists x_{n} \psi$, where $\psi\left(x_{1}, \ldots, x_{n}\right)$ is a finite conjunction of some atomic formulas, say $t_{1}=s_{1}, \ldots, t_{h}=s_{h}$, and some negations of atomic formulas, say $\psi_{1}, \psi_{2}, \ldots, \psi_{k}$. Assume, now, that $\psi$ is satisfied by $a_{1}, \ldots, a_{n}$ in some member $A$ of $V$.

Let $F\left(y_{1}, \ldots, y_{n}\right)$ be the free algebra of rank $n$ in $V$ and $f: F\left(y_{1} \cdots y_{n}\right) \rightarrow A$ be the homomorphism determined by $f\left(y_{i}\right)=a_{i}, i=1, \ldots, n$. Consider the congruence $\theta$ of $F\left(y_{1}, \ldots, y_{n}\right)$ generated by $h$ pairs $\left(u_{1}, v_{1}\right), \ldots,\left(u_{h}, v_{h}\right)$, where $f\left(u_{j}\right)=t_{j}^{A}(\vec{a}), f\left(v_{j}\right)=s_{j}^{A}(\vec{a}), j=1, \ldots, h$. Since $A \models \psi(\vec{a})$ it follows that $\theta \subseteq$ ker $f$. Then, there exists a homomorphism $g: F\left(y_{1} \cdots y_{n}\right) / \theta \rightarrow A$ such that $g\left(y_{i} / \theta\right)=a_{i}, i=1, \ldots, n$. Hence, $\psi\left(x_{1}, \ldots, x_{n}\right)$ is true in the finitely presented algebra $F\left(y_{1} \ldots, y_{n}\right) / \theta$ by interpreting the variables $x_{i}$ with $y_{i} / \theta$. 
When $A$ is the subdirect product of a family $\left\{A_{i}: i \in I\right\}$ there are $i_{1}, \ldots, i_{k} \in$ $I$ such that $A_{i_{s}}$ satisfies $\psi_{s}\left(a_{1}\left(i_{s}\right), \ldots, a_{n}\left(i_{s}\right)\right), s=1, \ldots, k$. Then, $\sigma$ holds in $A_{i_{1}} \times \cdots \times A_{i_{k}}$.

The following is the main theorem of this paper.

THEOREM 1. For every variety $V$ the following are equivalent. $V$.

(i) Every quasi-identity true in all finite members of $V$ is true in all members of

(ii) $V=S P P_{U}\left(V_{\text {fin }} \cap V_{S I}\right)$.

(iii) $V_{S I} \subseteq S P_{U}\left(V_{\text {fin }} \cap V_{S I}\right)$

(iv) $V$ is universally-finite.

(v) Every finitely presented member of $V$ is residually finite.

(vi) $V_{S I}$ is universally-finite.

(vii) Every quasi-identity true in all finite members of $V_{S I}$ is true in all members of $V_{S I}$.

Proof. (i) $\rightarrow$ (ii). By well-known facts (cf. [4 and 16, Chapter 31](i) implies that $V=S P P_{U}\left(V_{\text {fin }}\right)$. Hence $V=S P P_{U} S P\left(V_{\text {fin }} \cap V_{S I}\right)=S P P_{U}\left(V_{\text {fin }} \cap V_{S I}\right)$.

(ii) $\rightarrow$ (iii), (iii) $\rightarrow$ (vi). Simple.

(iv) $\rightarrow$ (i), (vi) $\rightarrow$ (vii). Trivial.

(vii) $\rightarrow$ (i). Since $V=S P\left(V_{S I}\right)$.

(v) $\rightarrow$ (iv). From Lemma 1 .

$(\mathrm{i}) \rightarrow(\mathrm{v})$. Let $A$ be a finitely presented algebra in $V$. Then, $A$ is isomorphic to $F\left(x_{1}, \ldots, x_{n}\right) / \theta$, where $F\left(x_{1}, \ldots, x_{n}\right)$ is the free algebra on some system of generators $x_{1}, \ldots, x_{n}$ and $\theta$ is a finitely generated congruence.

Let $R\left(v_{1}, \ldots, v_{n}\right)$ be the conjunction of all the defining relations of $A$, i.e. the conjunction of all equalities $t\left(v_{1}, \ldots, v_{n}\right)=s\left(v_{1}, \ldots, v_{n}\right)$, where every pair $\left(t\left(x_{1}, \ldots, x_{n}\right), s\left(x_{1}, \ldots, x_{n}\right)\right)$ belongs to a fixed finite system of generators for $\theta$. Take elements $a, b \in A$ with $a \neq b$ and consider the sentence

$$
\Phi: \exists v_{1} \ldots \exists v_{n}\left(R\left(v_{1}, \ldots, v_{n}\right) \wedge p\left(v_{1}, \ldots, v_{n}\right) \neq q\left(v_{1}, \ldots, v_{n}\right)\right),
$$

where $p\left(x_{1}, \ldots, x_{n}\right) / \theta=a, q\left(x_{1}, \ldots, x_{n}\right) / \theta=b$. But, sentence $\Phi$ is the negation of a quasi-identity which is true in $A$. Therefore, from (i) $\Phi$ must be true in a finite member $B$ of $V$. Hence, there exists a homomorphism $f_{a b}: A \rightarrow B$ with $f_{a b}(a) \neq f_{a b}(b)$. This proves $(\mathrm{v})$.

COROLLARY 1. Let $V$ be a variety such that every finitely generated subdirectly irreducible member of $V$ is finite, then every subvariety of $V$ is universally-finite.

We make some remarks on the results obtained and we discuss the most natural examples.

REMARKs 1.1. Let $A$ be a subdirectly irreducible commutative unitary ring generated by $n$ elements. Then, $A$ is the quotient of the polynomial ring $\mathbf{Z}\left[x_{1}, \ldots, x_{n}\right]$ by a completely irreducible, hence primary ideal $I$. We take a maximal ideal $M$ which contains $I$ and hence the prime ideal belonging to $I$. Then, from Theorem $12^{\prime}$ of $[\mathbf{2 1}$, p. 217], we can show that there exists some positive integer $k$ such that $M^{k}$ ( $I$. Therefore, $A$ must be finite since $\mathbf{Z}\left[x_{1}, \ldots, x_{n}\right] / M^{k}$ is finite.

Then, from ('orollary 1, any variety of commutative unitary rings is universallyfinite. 
1.2. If an element in a unitary ring has more than one left inverse, then it has infinitely many (I. Kaplansky). Therefore, the universal formula

$$
\forall x_{1} \forall x_{2} \forall x\left(x_{1} x=1 \wedge x_{2} x=1 \rightarrow x_{1}=x_{2}\right)
$$

is true in all finite unitary rings. But, such a formula is false in some rings with unity.

1.3. Locally finite or residually countable (cf. [20]) varieties are universally-finite by Corollary 1 .

1.4. Let $V$ be the variety of von Neumann commutative regular rings with quasi-inverse as operation. $V_{S I}$ is the class of all fields (cf. [18]) which is (cf. [19] a universally-finite class. Therefore, from Theorem $1, V$ is universally-finite.

1.5. An algebra $A$ is called Hopfian iff every onto endomorphism of $A$ is an automorphism. From Theorem 1, every finitely presented algebra of a universallyfinite variety is Hopfian (see [17, Lemma 6, p. 287]).

1.6. Every universally-finite variety is determined by its finite members. However, the converse is false.

Since every free group is residually finite (see $[\mathbf{1 3}]$ ) the variety $\mathcal{G}$ of groups is determined by the class of finite groups. But, $\mathcal{G}$ is not universally-finite since a Higman's example (see [11]) provides a finitely presented group which is nonHopfian.

From another Higman's example $[\mathbf{1 2}]$ it is possible to show that the universalHorn sentence

$$
\forall x \forall y \forall z \forall v\left(\left(y^{-1} x y=x^{2} \wedge z^{-1} y z=y^{2} \wedge v^{-1} z v=z^{2} \wedge x^{-1} v x=v^{2}\right) \rightarrow x=1\right)
$$

is true in all finite groups but it is false in some groups (see also [6, Lemma 6.1, p. 177]).

1.7. Since every finitely presented lattice is residually finite (see [8, p. 298]) the variety $\mathcal{L}$ of lattices is universally-finite. However there are some subvarieties of $\mathcal{L}$ (cf. [2]) which are not even determined by their finite members. This shows that a subvariety of a universally-finite variety is not necessarily universally-finite.

3. Reduction to equational theories. Let $T$ be a theory in any language. In this section using simple observations we show that Baldwin's problem for $T$ is reducible to the same problem for an appropriate equational theory associated with $T$.

We state first two easy propositions without proof. For the proof of the first proposition recall that a structure is finite if and only if it is isomorphic to each of its ultrapowers.

Proposition 1. Let $K$ and $K^{\prime}$ be classes of structures in languages $L$ and $L^{\prime}$, respectively. Suppose that $K$ and $K^{\prime}$ are closed under isomorphisms and ultraproducts. Consider $K$ and $K^{\prime}$ as categories with morphisms all monomorphisms. If there exist functors $F: K \rightarrow K^{\prime}$ and $G: K^{\prime} \rightarrow K$ which preserve ultraproducts and define an equivalence of categories, then, $K$ is universally-finite if and only if $K^{\prime}$ is universally-finite.

Proposition 2. Let $T$ be a theory in a language $L$. Then there exist a language without relation symbols $L^{\prime}$ and a theory $T^{\prime}$ in $L^{\prime}$ such that the category $K$ of models 
of $T$ and the category $K^{\prime}$ of models of $T^{\prime}$ are equivalent by functors which preserve ultraproducts.

For the remainder of this section we will assume that $L$ is a language without relation symbols and $d$ is a new symbol of a ternary operation; we denote the expanded language by $L^{d}$. If $T$ is a theory in $L$, we define the theory $T^{d}$ in $L^{d}$ by the axioms of $T$ plus

$$
\forall x \forall y \forall z(d(x, x, z)=z \wedge(x=y \vee d(x, y, z)=x)) .
$$

The models of $T^{d}$ are the models of $T$ with addition of a ternary discriminator. (For a comprehensive treatment of discriminator varieties see [4], Chapter IV, §9.) Finally, we denote the equational part of the theory $T^{d}$ by $\operatorname{Eq}\left(T^{d}\right)$.

We need also the following

DEFINITION. We say that a theory $T$ has the property of Embedding Reducts of Finite Submodels (ERFS) if every reduct $A$ to a finite type $\tau$ of a finite submodel of $T$ can be embedded in a reduct to $\tau$ of a finite model of $T$.

REMARK 3.1. Note that every universal theory has the property ERFS, but not the converse. The theory of fields presented in the language $\{+, \cdot, 0,1\}$ has the ERFS, but it is not universal.

PROPOSITION 3. Let $T$ be a first order theory in a language $L$ without relation symbols. $\mathrm{Eq}\left(T^{d}\right)$ denotes, as above, the set of equations in the language $L^{d}$ which are deducible by the theory $T$ plus axioms which guarantee that the new ternary operation symbol d becomes a discriminator on models of $T$. Then, the following are equivalent.

(i) $T$ is universally-finite.

(ii) $T$ has the property ERFS and the equational theory $\mathrm{Eq}\left(T^{d}\right)$ is universallyfinite.

ProOF. $K$ and $K^{d}$ denote the classes of models of $T$ and $T^{d}$, respectively. If $V$ is the variety of models of $\operatorname{Eq}\left(T^{d}\right)$, then our proposition follows by Theorem 1 and the following claims.

Claim 1. $T$ universally-finite implies that $T$ has the property ERFS.

Claim 2. $K$ is universally-finite iff $K^{d}$ is universally-finite.

Claim 3. $K^{d}$ universally-finite implies that $S K^{d}$ is universally-finite.

Claim 4. $S K^{d}$ universally-finite and the property ERFS for $T$ imply that $K^{d}$ is universally-finite.

Claim 5. $S K^{d}$ is universally-finite iff the class $V_{S I}$ is universally-finite.

PROOF OF ClAIM 1. Let $\tau$ be a finite subtype of the type for the language $L$. Suppose that $A$ is a reduct to $\tau$ of a finite submodel of $T$. Replace the constants in the conjunction of the diagram of $A$ by variables. We get a formula whose existential quantification is a sentence $\sigma$ that is true in a structure $B$ for the language $L$ if and only if $A$ can be embedded in the reduct of $B$ to $\tau$. But, when $T$ is universallyfinite $\sigma$ must be true in a finite model of $T$. The proof of Claim 2 is immediate by Proposition 1; proofs of Claims 3 and 4 are simple.

PROOF OF ClAIM 5. The discriminator variety $V$ is generated by the class $K^{d}$. Then, by well-known facts (cf. [4, Theorem 9.4, p. 165]) which follow from Jonsson's celebrated lemma (see [14]) the class $V_{S I}$ consists of the class $S K^{d}$ plus the one element algebra. To finish our proof observe that if the sentence 
$\forall z_{1} \cdots \forall z_{n} \psi\left(z_{1}, \ldots, z_{n}\right)$, where $\psi$ is an open formula, is true in all finite members of $S K^{d}$, then the universal sentence $\forall x \forall y \forall \vec{z}(x=y \vee \psi(\vec{z}))$ is true in all finite members of $V_{S I}$.

4. Universally-finite varieties of $R$-modules. In this section we derive other consequences of the main theorem about the variety $R$-Mod of left $R$-modules, where $R$ is a unitary ring.

COROLlARY 2. Let $R$ be a left Noetherian ring. Then, the following are equivalent.

(i) $R$-Mod is universally-finite.

(ii) Every cyclic subdirectly irreducible left $R$-module is finite.

(iii) $R$-Mod has a locally finite cogenerator.

Proof. (i) $\rightarrow$ (ii). Since $R$ is left Noetherian, every cyclic left $R$-module is finitely presented. Therefore, from (i) and Theorem 1 we get (ii).

(ii) $\rightarrow$ (iii). If $\left\{T_{i}: i \in I\right\}$ is a system of representatives of all simple left $R$ modules, then $C=\bigoplus_{i \in I} E\left(T_{i}\right)$ is a cogenerator of $R$-Mod (see [1, p. 211]). Here, $E(T)$ is the injective hull of $T$. Since every finitely generated submodule of $C$ is a finite sum of a finite number of cyclic subdirectly irreducible submodules of $C$, (ii) implies that $C$ is locally finite.

(iii) $\rightarrow$ (i). If $C$ is a cogenerator for $R$-Mod, then $R$-Mod $=S P\{C\}$. Therefore, from (iii) $R$-Mod satisfies (i) of Theorem 1 . Hence, we have (i).

COROLlARY 3. Let $R$ be a left Artinian ring, then the following are equivalent.

(i) $R$-Mod is universally-finite.

(ii) ${ }_{R} R$ as left $R$-module is residually finite.

(iii) $R$ is a finite ring.

Proof. (i) $\rightarrow$ (ii). From Theorem 1 .

(ii) $\rightarrow$ (iii). Since $R$ is left Artinian, ${ }_{R} R$ is finitely cogenerated (see [1, Proposition 10.18]). Hence, (ii) $\rightarrow$ (iii).

(iii) $\rightarrow$ (i). Obvious, since (iii) implies that $R$-Mod is locally finite.

COROLlARY 4. Let $R$ be a subring of the ring $S$ such that $R$ is a pure $R$ submodule of $S$. Then, $S$-Mod universally-finite implies that also $R$-Mod is so.

Proof. Let $A$ be a finitely presented $R$-module. Then, there exists an exact sequence $0 \rightarrow K \rightarrow R^{n} \rightarrow 0$ with $K$ finitely generated. Let $H$ be the $S$-submodule of $S^{n}$ generated by the generators of $K$. Since $R$ is a pure $R$-submodule of $S$, there exists an $R$-embedding of $A$ into $B=S^{n} / H$. Therefore, $A$ is residually finite in $R$-Mod because $B$ is residually finite in $S$-Mod.

COROLlARY 5. For every von Neumann commutative regular ring $R$ the following are equivalent.

(i) $R$-Mod is universally-finite.

(ii) $R$ is a subdirect product of finite fields.

Proof. (i) $\rightarrow$ (ii). From (i) $R$ is residually finite. Since every ideal in $R$ is the intersection of maximal ideals, it follows (ii).

(ii) $\rightarrow$ (i). Since $R$ is von Neumann regular, every finitely presented $R$-module $A$ is embeddable in a free module (cf. $[\mathbf{7}$, p. 10]). Therefore, from (ii) $A$ is residually finite. 
REMARKS. 4.1. Examples of rings $R$ where $R$-Mod is universally-finite are the following. By Corollary 2: every finite ring; every finitely generated commutative ring (see Remark 1.1); the ring $R$ of polynomial or formal power series in $n$ indeterminates over a finite field (see $\left[\mathbf{2 1}\right.$, Theorem $12^{\prime}$, p. 217]); every ring $R$ which is Noetherian and algebraically compact (see $[\mathbf{1 0}$, Lemma 5.6, p. 70]). By Corollary 5: every Boolean ring.

4.2. For every ring $R$ condition (ii) of Corollary 2 is sufficient for $R$-Mod to be universally-finite; however, it is not always necessary.

Let $R$ be a cartesian product of infinitely many pairwise nonisomorphic finite fields. By Corollary $5 R$-Mod is universally-finite. But, $R$ has as quotient an infinite field $F$ that is a simple $R$-module; hence, $F$ is an infinite cyclic subdirectly irreducible $R$-module.

4.3. In Corollary 4 we cannot assert that $R$-Mod is universally-finite if and only if $S$-Mod is so. Take as a counterexample a finite field $R$ and an infinite field $S$ which extends $R$.

4.4. Recall that every ring $R$ is Morita equivalent to the $n \times n$ matrices ring $M_{n}(R)$ for every $n$ (see $[1$, Corollary 22.6, p. 265]), i.e. the categories $R$-Mod and $M_{n}(R)$-Mod are equivalent. Moreover, the category Mod- $R$ of right $R$-modules is equivalent to the category $R$-Mod of left $R$-modules. Observe that functors which define an equivalence between varieties considered as categories must preserve ultraproducts. Then, from Proposition 1, Mod- $R$ and $M_{n}(R)$-Mod for every natural number $n$ are universally-finite when $R$-Mod is universally-finite.

PROBLEMS. 1. Let $V$ be a congruence distributive variety. Is $V$ universally-finite iff $V$ is determined by its finite members?

2. Describe the class $K$ of unitary rings $R$ such that $R$-Mod is universally-finite. In particular, prove or disprove:

(a) $K$ is closed under subrings.

(b) $K$ is closed under cartesian products.

(c) $R \in K, R$ commutative does imply $R\left[x_{1}, \ldots, x_{n}\right], R\left[\left[x_{1}, \ldots, x_{n}\right]\right] \in K$.

(d) $R \in K$ iff $R$ is residually finite.

Note that a positive answer to (d) would imply a positive answer to (a), (b), (c).

\section{REFERENCES}

1. F. W. Anderson and K. R. Fuller, Rings and categories of modules, Graduate Texts in Math., no. 13, Springer-Verlag, Berlin and New York, 1973.

2. K. A. Baker, Equational classes of modular lattices, Pacific J. Math. 28 (1969), 9-15.

3. J. T. Baldwin, Review. Selected papers of Abraham Robinson. Volume 1, J. Symbolic Logic 47 (1982).

4. S. Burris and H. P. Sankappanavar, A course in universal algebra, Graduate Texts in Math., no. 78, Springer-Verlag, Berlin and New York, 1981.

5. C. C. Chang and H. J. Keisler, Model theory, Studies in Logic, North-Holland, Amsterdam, 1973.

6. U. Felgner, The model theory of FC-groups, Mathematical Logic in Latin America (Arruda, Chuaqui and da Costa, Eds.), North-Holland, Amsterdam, 1980.

7. K. R. Goodearl, Ring theory, Pure and Appl. Math. 33, Marcel Dekker, New York, 1976.

8. G. Grätzer, General lattice theory, Mathematische Reihe 52, Birkhäuser-Verlag, Basel, 1978.

9. ___ Universal algebra, 2nd ed., Springer-Verlag, Berlin and New York, 1979.

10. D. K. Haley, Equational compactness in rings, Lecture Notes in Math., vol. 745, Springer-Verlag, Berlin and New York, 1979. 
11. G. Higman, A finitely related group with an isomorphic proper factor group, J. London Math. Soc. 26 (1951), 59-61.

12. __ A finitely generated infinite simple group, J. London Math. Soc. 26 (1951), 61-64.

13. Iwasawa Kenkiti, Einige Sätze über freie Gruppen, Proc. Imp. Acad. Tokyo 19 (1943), 272-274.

14. B. Jónsson, Algebras whose congruence lattice are distributive, Math. Scand. 21 (1967), 110-121.

15. D. W. Kueker, $A$ note on the elementary theory of finite abelian groups, Algebra Universalis 3 (1973), 156-159.

16. A. I. Mal'cev, The metamathematics of algebraic systems, Collected Papers 1936-1967, NorthHolland, Amsterdam, 1971.

17. __ Algebraic systems, Die Grundlehren der Math. Wissenshaften, vol. 192, Springer-Verlag, Berlin and New York, 1973.

18. R. S. Pierce, Modules over commutative regular rings, Mem. Amer. Math. Soc. No. 70 (1967).

19. A. Robinson, On axiomatic systems which possess finite models, Methods 3 (1951), 140-149.

20. W. Taylor, Residually small varieties, Algebra Universalis 2 (1972), 33-53.

21. O. Zariski and P. Samuel, Commutative algebra, Vol. 1, Graduate Texts in Math., No. 28, Springer-Verlag, Berlin and New York, 1977.

22. S. Burris and H. Werner, Sheaf constructions and their elementary properties, Trans. Amer. Math. Soc. 248 (1979), 269-309.

23. R. McKenzie, On spectra, and the negative solution of the decision problem for identities having a finite nontrivial model, J. Symbolic Logic 40 (1975).

Department of MATHEMATiCs AND Physics, UNiversity of CAMERino, 62032 CAMERINO, ITALY 